\title{
The Cytotoxic T Lymphocyte Antigen-4 +49A/G Single Nucleotide Polymorphism Association With Visceral Leishmaniasis
}

\author{
Mehrdad Hajilooi ${ }^{1}$; Pegah Lotfi ${ }^{2}$; Farhad Seif $^{3}$; Ahad Bazmani ${ }^{4}$; Mohammad Momeni ${ }^{5}$; Ali \\ Ravary ${ }^{6}$; Mohammad Kazemi Arababadi ${ }^{5}$; Ali Reza Khalilian ${ }^{7, *}$ \\ ${ }^{1}$ Department of Immunology, Faculty of Medicine, Hamadan University of Medical Sciences, Hamadan, IR Iran \\ ${ }^{2}$ Department of Microbiology, Faculty of Medicine, Hamadan University of Medical Sciences, Hamadan, IR Iran \\ 3 Department of Immunology and Microbiology, Faculty of Medicine, Shahrekord University of Medical Sciences, Shahrekord, IR Iran \\ ${ }_{5}^{4}$ Infectious and Tropical Diseases Research Center, Tabriz University of Medical Sciences, Tabriz, IR Iran \\ 5 Infectious and Tropical Diseases Research Center, Tabriz University of Medical Sciences, Tabriz, IR Iran \\ ${ }_{6}$ Department of Immunology, School of Public Health, Tehran University of Medical Sciences, Tehran, IR Iran
6 Occupational Environment Research Center, Rafsanjan University of Medical Sciences, Rafsanjan, IR Iran \\ 7 Faculty of Medicine, Shahid Beheshti Hospital, Hamadan University of Medical Sciences, Hamadan, IR Iran \\ ${ }^{*}$ Corresponding author: Ali Reza Khalilian, Shahid Beheshti Hospital, Faculty of Medicine, Hamadan University of Medical Sciences, Hamadan, IR Iran. Tel: +98-8118271560, Fax: +98- \\ 8118254073, E-mail: immunology86@yahoo.com
}

Received: June 10, 2013; Revised: Augest 04, 2013; Accepted: September 10, 2013

Background: Several lines of evidence approve that innate and adaptive immunity play key roles in the defense against visceral leishmaniasis (VL). The polymorphism within the cytotoxic T lymphocyte antigen 4 (CTLA-4) gene alters its expression.

Objectives: The main aim of this study was to evaluate the polymorphism within the +49 position of the CTLA- 4 gene of Iranian patients with VL in comparison with healthy controls.

Materials and Methods: In this cross-sectional study, 88 patients with clinical presentations of VL, who were seropositive for Leishmania (group 1), 86 patients without clinical presentations but seropositive (group 2), and 115 healthy controls (group 3) were assessed with respect to the CTLA-4+49A/G polymorphism, using polymerase chain reaction-restriction fragment length polymorphism (PCR-RFLP). The anti-Leishmania antibody titration was evaluated using an immunofluorescence method.

Results: Our results indicated that both CTLA-4 $+49 \mathrm{~A} / \mathrm{G}$ polymorphisms were significantly associated with VL.

Conclusions: According to the results, the polymorphisms within the +49 position of CTLA-4 can be associated with VL and may be considered as risk factors for the disease.

Keywords: Visceral Leishmaniasis; CTLA-4; Polymorphism

\section{Background}

Several Leishmania species are responsible for leishmaniasis, a vector-borne parasitic disease (1). Previous investigations demonstrated that although the sand fly vector is the main cause of Leishmania transmission into humans (1), the parasite can also be transmitted via blood transfusions (2), needlestick injury (3), or the mother/child route during pregnancy (4). Various clinical manifestations, including cutaneous, mucocutaneous, and visceral forms of the disease (5) are plausible after Leishmania infection, among which the visceral form (also known as Kala Azar) is the most severe form that can be lethal if left untreated (6). The quality and quantity of host immune system against the infection determines the final clinical outcome and severity of the disease (7).

$\mathrm{T}$ lymphocytes play key roles in combating against leishmaniasis via cytokine production (T-helper cells) and killing the parasite (8). Cytotoxic T lymphocyte antigen 4 (CTLA-4) is expressed on activated $\mathrm{T}$ cells and interacts with CD80 and CD86, which also are ligands for CD28, resulting in inhibition of $\mathrm{T}$ lymphocytes functions
(9). Based on its important roles in T lymphocyte suppression, it appears that CTLA-4 plays key roles in inducing peripheral tolerance against self and foreign antigens, which are seen in self-tolerance and chronic infections, respectively. Therefore, genetic variations that lead to up-regulation of this molecule can be associated with immune suppression and chronic infections, including visceral leishmaniasis (VL). Previous studies showed that the CTLA-4 gene contained several polymorphisms including CTLA-4 A/G and T/C at the +49 position (reference single nucleotide polymorphisms (SNP): (rs) 231775) (10). Additionally, CTLA- $4+49$ A allele resulted in increase of CTLA-4 expression $(11,12)$. Hence, it appears that this allele increases the inhibitory function of CTLA-4 on T cell proliferation and leads to attenuated immune responses against infections.

\section{Objectives}

Due to the fact that the quality and quantity of immune responses identify the outcome of leishmaniasis, the

Copyright (C) 2014,Ahvaz Jundishapur University of Medical Sciences; Published by Kowsar. This is an open-access article distributed under the terms of the Creative Commons Attribution-NonCommercial 4.0 International License (http://creativecommons.org/licenses/by-nc/4.0/) which permits copy and redistribute the material just in noncommercial usages, provided the original work is properly cited. 
main aim of this study was to evaluate the CTLA- $4+49 \mathrm{~A} / \mathrm{G}$ polymorphism in seropositive patients with VL with and without clinical presentation, in comparison with healthy controls.

\section{Materials and Methods}

\subsection{Subjects}

In this cross-sectional study, 88 patients with VL with antibody against Leishmania (seropositive) (group 1), 86 seropositive healthy controls (group 2), and 115 seronegative healthy controls (group 3) were recruited for sample donation. VL was diagnosed by an expert specialist according to laboratory findings, medical histories, and clinical presentations. The patients with $\mathrm{VL}$ as well as both groups 2 and 3 were selected from Meshkin city (north-west of Iran), where Leishmania infantum is endemic (13-15), while other species have not been reported. The Ethical Committee of the Ardabil University of Medical Sciences approved the study protocol and the participants filled out informed consent forms prior to entrance in the study.

\subsection{DNA Extraction}

Genomic DNA was purified from peripheral blood samples, which were obtained from the participants in ethylene diamine tetra acetic acid (EDTA) pretreated tubes, using a commercial kit from Bioneer Company (South Korea) according to the manufacturer's instructions.

\subsection{Polymorphism Detection}

The CTLA-4 +49A/G gene polymorphism was examined by the polymerase chain reaction-restriction fragment length polymorphism (PCR-RFLP) method. PCR was performed in a final volume of $20 \mu \mathrm{L}$, containing $0.3 \mu \mathrm{L}$ Taq DNA polymerase ( $5 \mathrm{U}), 2 \mathrm{Ml}$ Taq DNA polymerase buffer (10x), $2 \mu \mathrm{L}$ of each primer ( $25 \mathrm{ng} / \mu \mathrm{L}), 1 \mu \mathrm{L}$ of each dNTP [(dATP, dCTP, dGTP, dTTP) at a concentration of $10 \mathrm{mM}$ ] $2 \mu \mathrm{L}$ $\mathrm{MgCl}_{2}$ (stock concentration: $1.5 \mathrm{mM}$ ), $1 \mu \mathrm{L}$ of prepared DNA and sterile double distilled DNase free water. All the materials were purchased from Cinna Clone company (Iran). Sequences of forward and reverse primers (Cinna Clone, Iran) for amplification of the CTLA-4 +49A/G-containing region (162 bp) were 5'- GCTCTACTTCCTGAAGACCT-3' and 5'- AGTCTCACTCACCTTTGCAG-3', respectively. The following program was used for PCR amplifications: $95^{\circ} \mathrm{C}$ for five minutes (denaturation), followed by 35 cycles of 30 seconds at $95^{\circ} \mathrm{C}, 53^{\circ} \mathrm{C}$ for 30 seconds, and $72^{\circ} \mathrm{C}$ for 40 seconds, using a thermal cycler (Bioneer, South Korea).

Bbvi restriction enzyme (Cinna Clone, Iran) was obtained from Fermentas Company (Finland) and was used to recognize the polymorphism at the +49 position. Bbvl digested the PCR products of CTLA-4 +49A/G-containing position (162 bp), in the case of G allele, into $91 \mathrm{bp}$ and 71 bp sub-fragments. RFLP was performed according to the manufacturer's guidelines. Briefly, $2 \mathrm{U}$ of BbvI restriction enzyme and $5 \mu \mathrm{L}$ of its associated buffer were added to $10 \mu \mathrm{L}$ of the PCR products and incubated for 16 hours in room temperature. A $2.5 \%$ pretreated ethidium bromide agarose gel (CinnaClone, Iran) was used for electrophoresis of the PCR products and RFLP digestion products.

\subsection{Immun of Luorescence Assay}

The titration of the anti-Leishmania antibody was analyzed using a commercial kit from QIAGEN Company (USA), according to the manufacturer's guidelines. Briefly, a serial dilution of each participant's serum was prepared and the latest reacted concentration was determined as the anti-Leishmania titration.

\subsection{Statistical Analysis}

Hardy-Weinberg equilibrium analysis was used for evaluating the validity of the data. $\chi^{2}$ test was used from the SPSS software version 13 to identify the differences between the groups and P value less than 0.05 was considered significant.

\section{Results}

Our results indicated that CTLA- $4+49 \mathrm{~A} / \mathrm{G}$ polymorphism was significantly associated with VL $(\mathrm{P}<0.001)$. The results from the PCR-RFLP analysis demonstrated that the CTLA-4 +49-A/G genotype significantly increased in group 1 compared to both groups 2 and 3 . The results in detailed are presented in Table 1 and Table 2. The statistical analysis demonstrated that the differences between the groups regarding the genotypes and alleles were significant (Tables 1 and 2). The current results also demonstrated that the patients with VL (group 1) carrying the CTLA-4 +49-A/G genotype had higher mean anti-Leish mania antibody titers; the statistical analysis revealed that the difference was significant (Table 3$)(\mathrm{P}<0.001)$. The results also demonstrated that the titration of antiLeishmania antibody was significantly higher in group 3 participants carrying the CTLA-4 +49-A/G genotype than CTLA-4 +49-A/A and G/G genotypes (Table 3 ) $(\mathrm{P}<0.001$ ).

Table 1. The Prevalence of CTLA-4 +49A/G Polymorphism Among Patients With Clinical Presentation of VL who Were Seropositive for Leishmania (Group 1), Without Clinical Presentation but Seropositive (Group 2), and healthy controls (Group 3) ${ }^{\mathrm{a}, \mathrm{b}}$

\begin{tabular}{lccc}
\hline & Group 1 & Group 2 & Group 3 \\
\hline Genotypes & & & \\
$\mathrm{A} / \mathrm{A}$ & $33(37.5)$ & $52(60.5)$ & $48(41.7)$ \\
$\mathrm{A} / \mathrm{G}$ & $48(54.5)$ & $22(25.6)$ & $22(19.1)$ \\
$\mathrm{G} / \mathrm{G}$ & $7(8)$ & $12(14)$ & $45(39.1)$ \\
Alleles & & & \\
$\mathrm{A}$ & $114(64.8)$ & $126(73.3)$ & $118(51.3)$ \\
$\mathrm{G}$ & $62(35.2)$ & $46(26.7)$ & $112(48.7)$ \\
\hline
\end{tabular}

a Abbreviations: A, adenine; G, guanidine.

$\mathrm{b}$ Data are presented as No.\% 
Hajilooi M et al.

\begin{tabular}{|c|c|c|c|c|}
\hline \multirow[t]{2}{*}{ Genotypes } & \multicolumn{2}{|c|}{ Group 1 vs Group 2} & \multicolumn{2}{|c|}{ Group 1 vs Group 3} \\
\hline & OR $(95 \% \mathrm{CI})$ & P Value & OR $(95 \% \mathrm{CI})$ & P Value \\
\hline A/G-G/G & $3.74(1.29-10.79)$ & 0.013 & $14.02(5.46-36)$ & $\mathrm{P}=0.0001$ \\
\hline A/A-G/G & $1.09(0.38-3.04)$ & 0.87 & $4.42(1.77-10.99)$ & $P=0.001$ \\
\hline A/A-A/G & $0.29(0.15-0.56)$ & 0.0001 & $0.31(0.16-0.61)$ & $P=0.001$ \\
\hline
\end{tabular}

a Abbreviations: A, adenine; G, guanidine; vs, versus.

Table 3. Anti-Leishmania Antibody Titration in Groups 1 and 2 Carrying Various CTLA-4 +49 Genotypes a,b,c,d

\begin{tabular}{lcccc}
\hline & \multicolumn{3}{c}{ Anti-Leishmania Antibody Titration } & PValue \\
\cline { 2 - 4 } & CTLA-4 +49-A/A & CTLA-4 +49-A/G & CTLA-4 +49-G/G & \\
\hline Group 1 & $2.3 \pm 0.273^{\mathrm{e}}$ & $4.98 \pm 0.216$ & $1.86 \pm 0.508$ & $\mathrm{P}<0.001$ \\
Group 2 & $1.88 \pm 0.14^{\mathrm{f}}$ & $3.32 \pm 0.24$ & $0.75 \pm 0.15$ & $\mathrm{P}<0.001$ \\
\hline
\end{tabular}

${ }^{\mathrm{a}}$ Sincegroups 1 and 2 had anti-Leishmania antibody, the relationship between the anti-Leishmania antibody titration and CTLA-4 +49 genotypes were performed within groups 1 and 2 .

b Data are presented as labeled mean \pm standard error.

${ }^{\mathrm{C}}$ The titrations were labeled with the following numbers: 0 : no antibody, 1:1/10, 2:1/20, 3:1/40, 4:1/80, 5:1/160, and 6:1/320.

d Group 1, seropositive patients with VL; group 2, seropositive healthy controls.

e The differences between CTLA-4 +49-A/G and A/A genotypes were significant.

$\mathrm{f}$ The differences among CTLA-4 +49-A/A, A/G and G/G genotypes were significant.

\section{Discussion}

CTLA-4 is expressed on activated T lymphocytes and binds to CD80 and CD86, which results in suppression of the T lymphocyte function (16). Ectopic up-regulation of CTLA-4 during infectious diseases results in impaired immune responses and consequently induces chronic forms of infectious diseases (17). Previous studies demonstrated that expression of CTLA- 4 was regulated by the polymorphisms within the exon 1 ( +49 position) region of the CTLA-4 gene $(11,12)$. Our results showed that either genotypes or alleles of CTLA- $4+49 \mathrm{~A} / \mathrm{G}$ polymorphism were significantly associated with VL in the Iranian population. Additionally, the current results revealed that the CTLA-4 +49-A/G genotype was significantly increased in group 1 compared to groups 2 and 3 . As mentioned in the introduction section, the CTLA- $4+49$ A allele was associated with higher expression of CTLA-4; hence, it seems that higher prevalence of CTLA-4 +49-A/G genotype in Iranian patients with VL may be responsible for impaired immune responses against Leishmania in patients with VL.

Previous studies demonstrated that CTLA-4 plays a crucial role in suppression of immune responses against Leishmania during VL (18). Elevation levels of CTLA-4 in patients with VL were also reported by Katara and colleagues (19). Studies on animal models also revealed that CTLA-4 suppresses the immune responses against Leishmania via inducing the transforming growth factor- $\beta$ (TGF- $\beta$ ), an anti-inflammatory cytokine (20). Murphy et al. showed that using anti-CTLA-4 antibody for blocking CTLA-4 led to enhanced host resistance to L. donovani (21). Murray and colleagues also identified that using antiCTLA-4 antibody in L. donovani viscerally infected animals led to parasite killing by the immune cells (22). Another study by Saha et al. demonstrated that CTLA-4 may play key roles in regulation of T-helper 1/T-helper 2 balance, which is important to determine the leishmaniasis outcome (23). Another study by Zubairi and colleagues revealed that immunotherapy with anti-CTLA-4 increased the immune responses, which resulted in killing L. donovani (24). Therefore, based on the aforementioned studies, it seems that overexpression of CTLA-4 can be considered as a main reason for impaired immune responses, in which, different CTLA- $4+49$ genotypes may be responsible for up-regulation of this molecule in VL.

To the best of our knowledge this was the first study evaluating the CTLA- $4+49 \mathrm{~A} / \mathrm{G}$ polymorphism in leishmaniasis. However, there have been several studies on other chronic infections. For example, Danilovic et al. demonstrated that CTLA- $4+49 \mathrm{~A} / \mathrm{G}$ polymorphism was significantly associated with chronic hepatitis $C$ infection (25). Duan and colleagues reported that patients with chronic HBV infection had higher frequencies of CTLA-4 +49-A/A genotype and also A allele (26). Raitala et al. evaluated the activity of Helicobacter pylori (HP), inducing indoleamine 2,3-dioxygenase, in patients with chronic HP infections and found that the activity significantly increased in patients carrying CTLA-4 +49-A/A genotype (17). Therefore, based on the aforementioned study, CTLA- $4+49-\mathrm{A} / \mathrm{G}$ polymorphism can be significantly associated with chronic infections. These results confirmed the fact that the CTLA- $4+49 \mathrm{~A} / \mathrm{G}$ polymorphism plays a key role in VL pathogenesis.

Our results also showed that theCTLA- $4+49 \mathrm{~A} / \mathrm{G}$ polymorphism was significantly associated with anti-Leish- 
mania antibody titrations in groups 1 and 2 participants; hence, this polymorphism can be associated with antibody production against Leishmaniain Iranian patients. The results demonstrated that anti-Leishmania antibody titration significantly increased in either group1 or 2 with A/G genotype. Therefore, it appears that $A / G$ genotype not only can be considered as a risk factor for VL, but can be associated with higher antibody production against Leishmania, which may be related to shift immune responses of T-helper 2 immunity and consequently impaired cellular immunity. Finally, based on our results, it seems that the CTLA- $4+49 \mathrm{~A} / \mathrm{G}$ polymorphism is significantly associated with VL as well as antibody production against Leishmania in Iranian patients with VL.

\section{Acknowledgements}

We appreciate all patients and healthy controls, which participated in this research.

\section{Authors' Contributions}

All the authors were involved in all the steps of manuscript preparation.

\section{Funding/Support}

This project was supported by a grant from the Hamadan University of Medical Sciences.

\section{References}

1. Hartemink N, Vanwambeke SO, Heesterbeek H, Rogers D, Morley $\mathrm{D}$, Pesson $\mathrm{B}$, et al. Integrated mapping of establishment risk for emerging vector-borne infections: a case study of canine leishmaniasis in southwest France. PLoS One. 2011;6(8).

2. Wiwanitkit V. Bone marrow leishmaniasis: a review of situation in Thailand. Asian Pac J Trop Med. 2011;4(10):757-9.

3. Herwaldt BL, Juranek DD. Laboratory-acquired malaria, leishmaniasis, trypanosomiasis, and toxoplasmosis. Am J Trop Med Hyg. 1993;48(3):313-23.

4. Meinecke CK, Schottelius J, Oskam L, Fleischer B. Congenital transmission of visceral leishmaniasis (Kala Azar) from an asymptomatic mother to her child. Pediatrics. 1999;104(5).

5. Newlove T, Robinson M, Meehan SA, Pomerantz R. Old World cutaneous leishmaniasis. Dermatol Online J. 2012;18(12):32.

6. Wilson ME, Jeronimo SM, Pearson RD. Immunopathogenesis of infection with the visceralizing Leishmania species. Microb Pathog. 2005;38(4):147-60.

7. Ali N, Mekuria AH, Requena JM, Engwerda C. Immunity to visceral leishmaniasis. JTrop Med. 2012;2012:780809.

8. Santos Cda S, Boaventura V, Ribeiro Cardoso C, Tavares N, Lordelo MJ, Noronha A, et al. CD8(+) granzyme B(+)-mediated tissue injury vs. CD4(+)IFNgamma(+)-mediated parasite killing in human cutaneous leishmaniasis. J Invest Dermatol. 2013;133(6):1533-40.

9. Linsley PS, Brady W, Urnes M, Grosmaire LS, Damle NK, Ledbetter JA. CTLA-4 is a second receptor for the B cell activation antigen B7. J Exp Med.1991;174(3):561-9.
10. Uzer E, Dilmec F, Akkafa F, Boduroglu O, van Kuilenburg AB. Investigation of CTLA-4 and CD28 gene polymorphisms in patients with diabetes mellitus type 2 using PCR-RFLP in a Turkish population. West Indian Med J. 2010;59(3):235-40.

11. Anjos S, Nguyen A, Ounissi-Benkalha H, Tessier MC, Polychronakos C. A common autoimmunity predisposing signal peptide variant of the cytotoxic T-lymphocyte antigen 4 results in inefficient glycosylation of the susceptibility allele. J Biol Chem. 2002;277(48):46478-86.

12. Wang XB, Pirskanen R, Giscombe R, Lefvert AK. Two SNPs in the promoter region of the CTLA-4 gene affect binding of transcription factors and are associated with human myasthenia gravis. Intern Med. 2008;263(1):61-9.

13. Oshaghi MA, Ravasan NM, Javadian EA, Mohebali M, Hajjaran H, Zare Z, et al. Vector incrimination of sand flies in the most important visceral leishmaniasis focus in Iran. Am J Trop Med Hyg. 2009;81(4):572-7.

14. Oshaghi MA, Ravasan NM, Hide M, Javadian EA, Rassi Y, Sadraei J, et al. Phlebotomus perfiliewi transcaucasicus is circulating both Leishmania donovani and L. infantum in northwest Iran. Exp Parasitol. 2009;123(3):218-25.

15. Mohebali M, Javadian E, Yaghoobi-Ershadi MR, Akhavan AA, Hajjaran $\mathrm{H}$, Abaei MR. Characterization of Leishmania infection in rodents from endemic areas of the Islamic Republic of Iran. East Mediterr Health J. 2004;10(4-5):591-9.

16. Bachmann MF, Gallimore A, Jones E, Ecabert B, Acha-Orbea $\mathrm{H}_{\text {, }}$ Kopf M. Normal pathogen-specific immune responses mounted by CTLA-4-deficient T cells: a paradigm reconsidered. Eur J Immunol. 2001;31(2):450-8.

17. Raitala A, Karjalainen J, Oja SS, Kosunen TU, Hurme M. Helicobacter pylori-induced indoleamine 2,3-dioxygenase activity in vivo is regulated by TGFB1 and CTLA4 polymorphisms. Mol Immunol. 2007;44(5):1011-4.

18. Gomes NA, Gattass CR, Barreto-De-Souza V, Wilson ME, DosReis GA. TGF-beta mediates CTLA-4 suppression of cellular immunity in murine kalaazar.JImmunol. 2000;164(4):2001-8.

19. Katara GK, Ansari NA, Verma S, Ramesh V, Salotra P. Foxp3 and IL10 expression correlates with parasite burden in lesional tissues of post kala azar dermal leishmaniasis (PKDL) patients. PLoS Negl Trop Dis. 2011;5(5).

20. Goto H, Lindoso JA. Immunity and immunosuppression in experimental visceral leishmaniasis. Braz J Med Biol Res. 2004; 37(4):615-23.

21. Murphy ML, Cotterell SE, Gorak PM, Engwerda CR, Kaye PM. Blockade of CTLA-4 enhances host resistance to the intracellular pathogen, Leishmania donovani. JImmunol. 1998;161(8):4153-60.

22. Murray HW, Lu CM, Brooks EB, Fichtl RE, DeVecchio JL, Heinzel FP. Modulation of T-cell costimulation as immunotherapy or immunochemotherapy in experimental visceral leishmaniasis. Infect Immun. 2003;71(11):6453-62.

23. Saha B, Chattopadhyay S, Germond R, Harlan DM, Perrin PJ CTLA4 (CD152) modulates the Th subset response and alters the course of experimental Leishmania major infection. Eur J Immunol.1998;28(12):4213-20.

24. Zubairi S, Sanos SL, Hill S, Kaye PM. Immunotherapy with OX40LFC or anti-CTLA-4 enhances local tissue responses and killing of Leishmania donovani. Eur J Immunol. 2004;34(5):1433-40.

25. Danilovic DL, Mendes-Correa MC, Lima EU, Zambrini H, K. Barros R, Marui S. Correlations of CTLA-4 gene polymorphisms and hepatitis C chronic infection. Liver Int. 2012;32(5):803-8.

26. Duan S, Zhang G, Han Q, Li Z, Liu Z, Chen J, et al. CTLA-4 exon +49 polymorphism alone and in a haplotype with -318 promoter polymorphism may confer susceptibility to chronic HBV infection in Chinese Han patients. Mol Biol Rep. 2011;38(8):5125-32. 